\title{
Reduction of Common Mode Noise and Global Multi-valued Offset in Touch Screen Systems by Correlated Double Sampling
}

\author{
Shuo Gao, Student Member, IEEE, Jackson Lai, Charles Micou and Arokia Nathan, Fellow, IEEE
}

\begin{abstract}
Touch based interactivity has become an important function in displays. This paper reports on the signal processing of touch signals in which touch interactivity is processed as an image and correlated double sampling (CDS) algorithm is applied for both common-mode noise reduction and global multi-valued offset cancellation. Based on experimental results, we achieved a boost in SNR of 7.6dB. The processed signal reduces detection errors and power consumption of the system.
\end{abstract}

Index Terms-image signal processing, correlated double sampling, capacitance touchscreen, global multi-valued offset, common-mode noise.

\section{INTRODUCTION}

HE different types of touchscreen panels (TSPs) available in the market are resistive, capacitance, optical, acoustic and in-cell architectures [1], [2]. The two most widely used are the resistive and capacitive architectures. The former constitutes the first generation touchscreen [3], and is still being utilized in displays [4]. The latter is capacitance based and has the advantages of fast response, good visibility and multi-touch detection ability [2], [5]-[8].

Highly undesired in TSPs are detection errors and high power consumption, which lead to touch mis-registrations (in terms of presence and/or position) and short battery life. Two of the main factors related to these are the noise and the global multi-valued offsets.

As shown in Fig. 1, the noise in a TSP is generated from many sources. For example the charger induces common-mode noise including power supply spikes. Under these conditions, the signal-to-noise ratio (SNR) is compromised, causing the touch detection to fail and/or lead to "fake touch". In order to achieve a high SNR level, the normal way is to boost the excitation

Manuscript received xx x, 2015.

S. Gao, C. Micou and A. Nathan are with the Hetero-Genesys Laboratory, Department of Engineering, University of Cambridge, Cambridge CB3 0FA, U.K. (e-mail: sg690@cam.ac.uk; cm704@cam.ac.uk; an299@eng.cam.ac.uk).

J. Lai is with Display Technology Development, Blackberry Ltd., Mississauga, ON L4W 0B5, Canada (e-mail: jackson@laimail.ca).

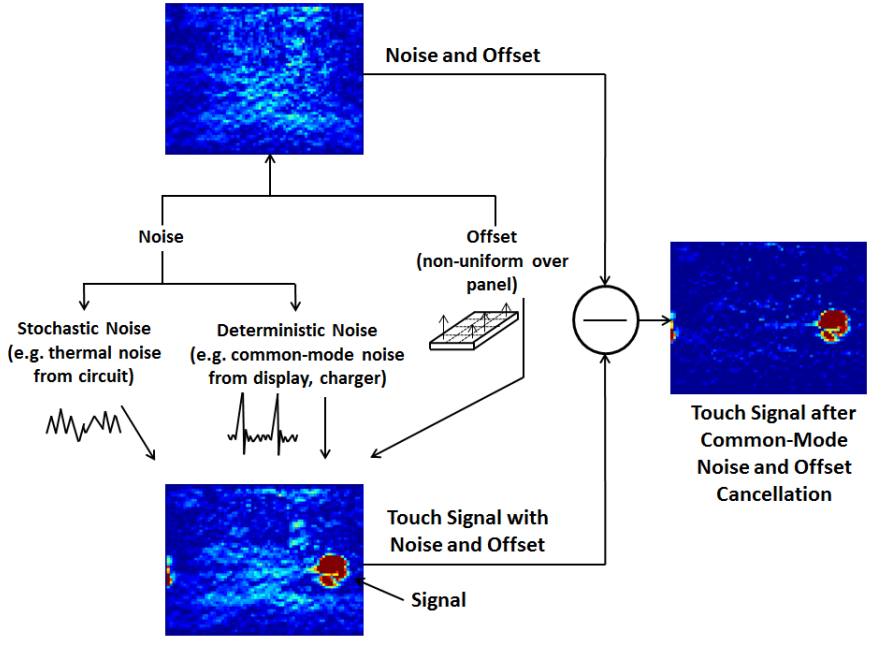

Fig. 1. Noise sources of touchscreen systems and concept of CDS algorithm. *Noise (or unwated signal) comprises of stochastic (thermal or $1 / f$ ) and determinstic (clock signal, power supply spike) components.

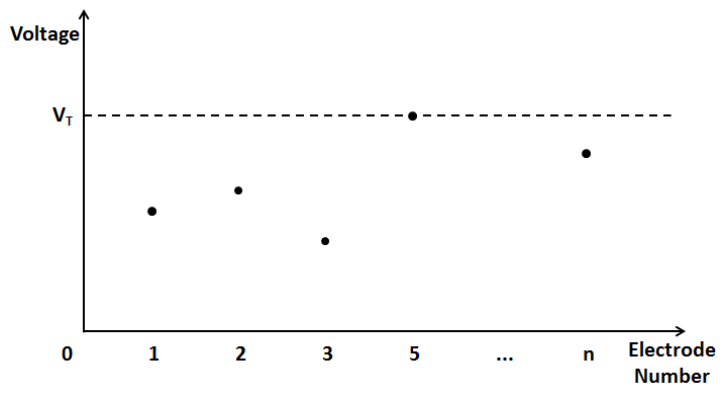

Fig. 2. The detection threshold $\left(V_{T}\right)$ is set by the highest offset value to avoid detection errors, resulting in increased power consumption.

power of the touch panel. However, this increases power consumption. Thus, reducing the noise in a TSP is critical for reduction of errors and the power consumption. In particular, in analog applications, such as force touch, in which the degree of force provides additional interactive functions, the noise alternately limits the resolution of force-touch.

The offset on a single electrode can be canceled by deducting a fixed value. However, the non-uniformity of the offsets over the whole panel makes it difficult, giving rise to a high threshold 


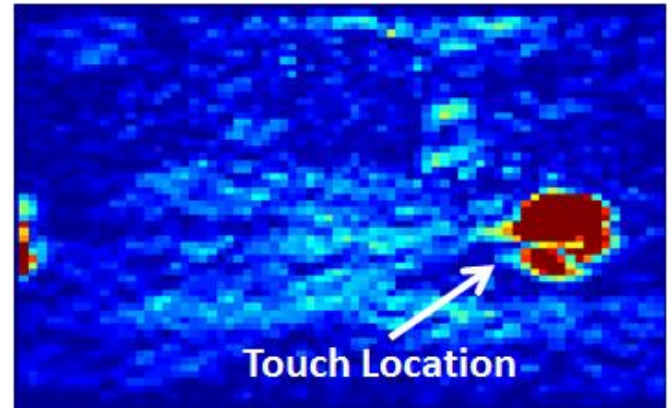

(a)

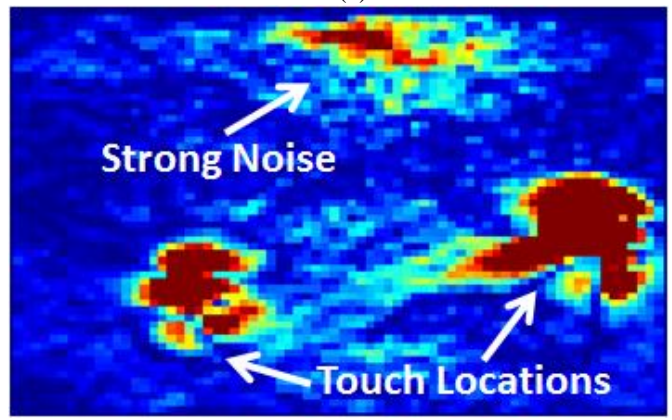

(b)

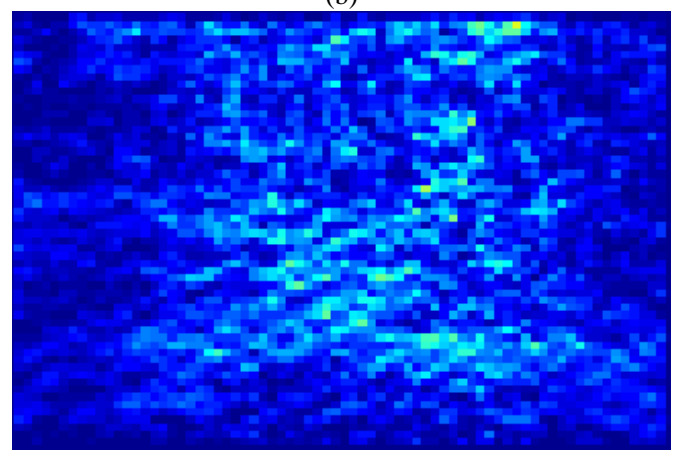

(c)

Fig. 3. Images of (a) single touch, (b) multi-touch, and (c) noise pattern. Red indicates high value region.

to avoid detection errors. As illustrated in Fig. 2, the detection threshold has to be set according to the highest offset voltage, thus requiring a high excitation power.

In this paper, a technique to reduce common-mode noise and global multi-valued offset is proposed and implemented. In the proposed technique, the data associated with the whole frame after each scanning action is treated as an image [9]-[11]. Each electrode intersection constitutes a pixel and provides a voltage value. In this way, image processing methods can be combined seamlessly with touch signal detection to remove any correlated noise and offset.

The touchscreen related images right after the single and multiple touch events are shown in Fig. 3. In an ideal case, only the pixels at the touch location have signal values larger than 0 . However, as shown in Fig. 3, almost all the pixels have positive values (i.e. non-blue), which indicate that the touch signal needs to be strong enough to overcome the noise. Here is where correlated double sampling (CDS) [12]-[33] can be used to

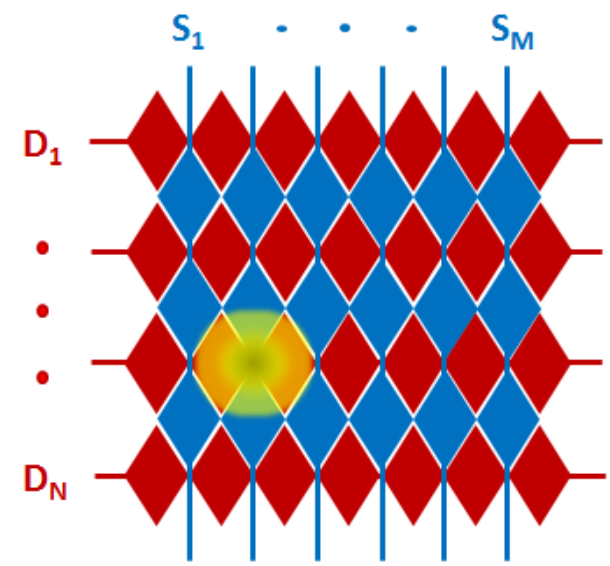

Fig. 4. Mutual-capacitance electrodes with diamond structure. Yellow region indicates the location of touch event.

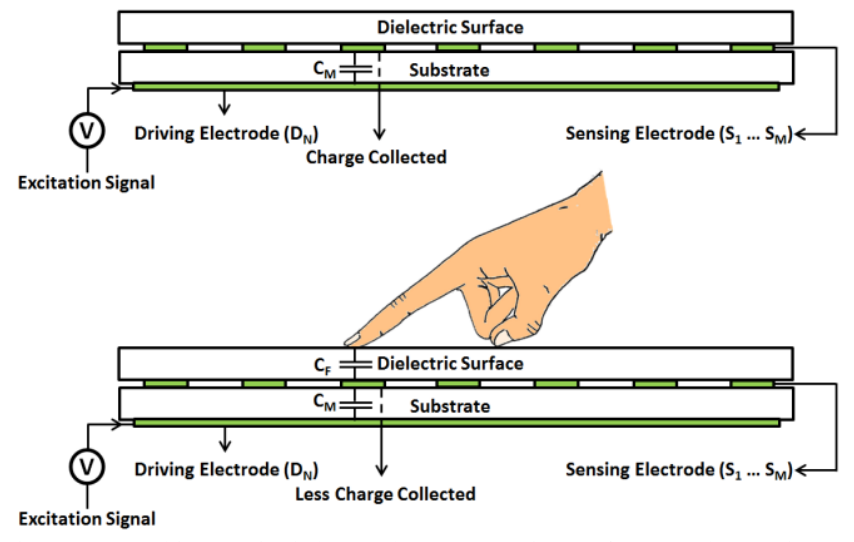

Fig. 5. Working principle and cross-section of mutual-capacitance touchscreen.

cancel the global multi-valued offset and common-mode noise (or fixed pattern noise as referred to in imaging terminology).

This paper is structured as follows. In Section II, a review of capacitance based touchscreens is given. In Section III, the relationship between CDS and SNR is theoretically analyzed. In Section IV, the parameters of the test bed are provided and the CDS based algorithm is presented. Discussion of experimental and simulation results is given in Section $\mathrm{V}$.

\section{II.CAPACITIVE TOUCHSCREENS}

The working principle of capacitance touchscreens is based on projected capacitance or surface capacitance. The latter is not widely used since it offers limited resolution and suffers from high noise due to parasitic capacitive coupling [4]. Projected capacitance measures the change of capacitance at electrodes to detect the touch event. When a conducting object (human finger or stylus) touches the screen, the electric field lines will be perturbed thus modulating the charge distribution and hence the capacitance. This is then sensed by electrodes and sent to the processor to determine the touch location. In projected capacitance, two options are provided here: self-capacitance and mutual capacitance. Since mutual 


\begin{tabular}{|c|c|c|c|c|c|c|c|c|}
\hline Ref. \# & [6] & [7] & [35] & [36] & [37] & [38] & [39] & [40] \\
\hline Multi-touch? & Yes & Yes & Yes & Yes & Yes & Yes & Yes & Yes \\
\hline Capacitance Type & Mutual & Self/Mutual & Mutual & Mutual & Mutual & Mutual & Mutual & Mutual \\
\hline Chip Area & $14.9 \mathrm{~mm}^{2}$ & $13.7 \mathrm{~mm}^{2}$ & $10.4 \mathrm{~mm}^{2}$ & $0.46 \mathrm{~mm}^{2}$ & $4 \mathrm{~mm}^{2}$ & $\begin{array}{c}\mathrm{TX}: 1.6 \mathrm{~mm}^{2} \\
\mathrm{RX}: 3.24 \mathrm{~mm}^{2}\end{array}$ & $2.2 \mathrm{~mm}^{2}$ & $6.9 \mathrm{~mm}^{2}$ \\
\hline Channels & $30 \times 24$ & $80 \times 80$ & $27 \times 43$ & $24 \times 16$ & $20 \times 16$ & $32 \times 20$ & $12 \times 8$ & $30 \times 24$ \\
\hline TSP Size & $5^{\prime \prime}$ & $4.5^{\prime \prime}$ & $10.1^{\prime \prime}$ & $4.8^{\prime \prime}$ & $7^{\prime \prime}$ & $10.1^{\prime \prime}$ & $4.3^{\prime \prime}$ & $3.2^{\prime \prime}$ \\
\hline Touch Controller & $\begin{array}{c}0.18 \mu \mathrm{m} \\
\mathrm{CMOS}\end{array}$ & $\begin{array}{c}0.35 \mu \mathrm{m} \\
\mathrm{CMOS}\end{array}$ & $\begin{array}{c}0.35 \mu \mathrm{m} \\
\mathrm{CMOS}\end{array}$ & $\begin{array}{c}0.18 \mu \mathrm{m} \\
\mathrm{CMOS}\end{array}$ & $\begin{array}{c}0.18 \mu \mathrm{m} \\
\mathrm{CMOS}\end{array}$ & $\begin{array}{c}0.18 \mu \mathrm{m} \\
\mathrm{CMOS}\end{array}$ & $\begin{array}{l}0.18 \mu \mathrm{m} \\
\mathrm{CMOS}\end{array}$ & $\begin{array}{l}90 \mathrm{~nm} \\
\text { triple well LDI }\end{array}$ \\
\hline
\end{tabular}

Table I. Current state-of-the-art of capacitive TSPs.

capacitance supports multi-touch and is less sensitive to electromagnetic interference (EMI) [34], it is investigated here, based on which experimental results are presented.

In mutual capacitance, the electrodes are normally placed as rows and columns. Electrodes in rows work as driving lines and those in columns act as sensing lines, or vice versa. Each intersection of rows and columns indicates a unique location, and each intersection can be measured individually, thus multi-touch is supported. As shown in Fig. 4 (front view) and Fig. 5 (cross-section view), electrodes in rows are arranged as $D_{1}$ to $D_{N}$, after each of them is powered separately, the intersections with the sensing lines from $S_{1}$ to $S_{M}$ are measured in sequence to realize multi-touch detection. As depicted in Fig. 5 , before the finger touch, the mutual capacitance is $C_{M}$. During the touch, the capacitance $C_{F}$ is created between the electrode and human finger. One drawback of this sensing method is that more time is needed for a full screen measurement as compared with that of self-capacitance. Current touchscreens have a sensing frame rate from $20 \mathrm{~Hz}$ to $200 \mathrm{~Hz}$ [4].

Many noise reduction and sensing schemes have been proposed and implemented. A summary of the state-or-the-art in capacitance TSPs is shown in Table I.

\section{THEORETICAL ANALYSIS}

Signal-to-noise ratio is widely used to represent a system's detection accuracy and resolution. The SNR is defined as the ratio of the signal power $\left(P_{s}\right)$ to the noise power $\left(P_{n}\right)$ :

$$
S N R=\frac{P_{s}}{P_{n}}
$$

whereby SNR>1 implies that the touch signal surpasses the noise. The offset is not dealt as a noise component, as it can be removed by deducting a fixed value. As depicted in Fig. 6(a), the output from an electrode of a capacitance based TSP consists of the touch signal, the offset, and noise or unwanted signal. The offset together with common-mode noise can be canceled by CDS as shown in Fig. 6(b), leaving signal and high frequency noise. After CDS, the SNR is expressed as:

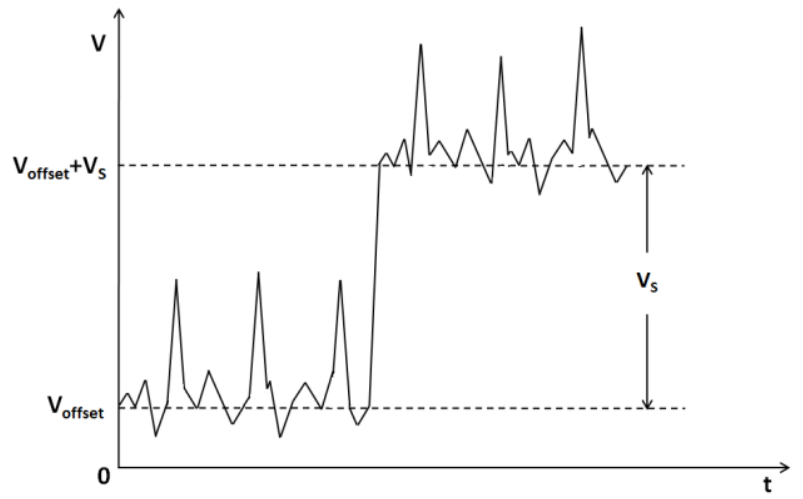

(a)

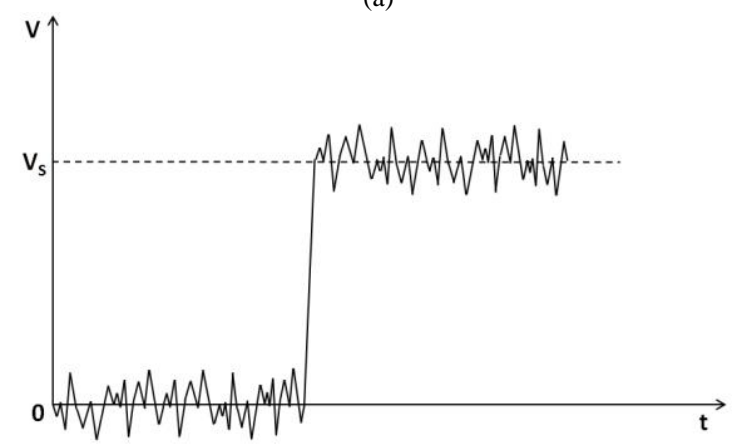

(b)

Fig. 6. Conceptual outputs from an electrode of mutual capacitance TSP without and with touch. (a) Original output comprising offset and common-mode noise, and (b) the output after CDS. $\mathrm{V}_{\mathrm{S}}$ is the signal voltage and $\mathrm{V}_{\text {offset }}$ is the offset voltage.

$S N R_{C D S}=\frac{P_{s}}{P_{n}{ }^{\prime}}=\frac{P_{s}}{P_{n} / \alpha} ;$

where $S N R_{C D S}$ represents the SNR after CDS, $P_{n}$ ' the noise power after CDS, and $\alpha$ the ratio of $P_{n}$ to $P_{n}$ '. The range of $\alpha$ values and corresponding implications are given in Table II. In the ideal case, if two samples are obtained at the same time, the common-mode noise can be canceled completely. Thus it seems that faster sampling can provide higher SNR. However, this is not always true in practice. For example, if a single frequency 


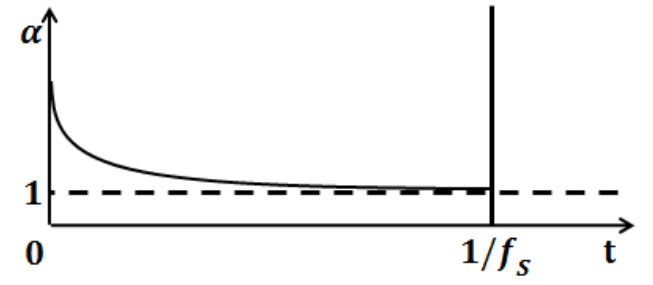

Fig. 7. Conceptual relationship between characterization factor $\alpha$ and sampling interval.

\begin{tabular}{|c|c|c|}
\hline Range & SNR & Example \\
\hline $0<\alpha<1$ & SNR Degrades & ${ }_{f_{S}=2 f_{H}}{ }_{f_{1}}{ }^{1}$ \\
\hline$\alpha=1$ & SNR Maintained & $\overbrace{2 f_{H}>f_{S}>f_{H}}^{-}$ \\
\hline $1<\alpha<+\infty$ & SNR Boosted & $\underbrace{}_{f_{S} \gg f_{H}}$ \\
\hline
\end{tabular}

Table II. The ranges of characterization factor $\alpha$ and corresponding implications. $f_{S}$ and $f_{H}$ are the sampling frequency and waveform frequency, respectively. In reality, $f_{H}$ is the highest noise frequency within a system's noise bandwidth. If the system is a one-pole low-pass filter, $f_{H}=(\pi / 2) f_{3 d B}$, where $f_{3 d B}$ is system's $-3 \mathrm{~dB}$ bandwidth.

\begin{tabular}{ccc}
\hline \hline Parameter & Unit & Value \\
\hline Diagonal & Inch & 10.1 \\
Aspect Ratio & None & $16 / 9$ \\
Excitation Voltage & Volt & 10 \\
Display Pixel Size & (Micrometer) $^{2}$ & $56 \times 56$ \\
TX Electrode Size & Millimeter & 3 \\
RX Electrode Size & Micrometer & 449 \\
Refreshing rate & Hertz & 60 \\
Sensing Array Size & (Millimeter) $^{2}$ & $3 \times 3$ \\
Sensing Array Spacing & Millimeter & 2 \\
\hline \hline
\end{tabular}

Table III. Parameters of the test bed.

waveform is considered, faster sampling doesn't imply higher SNR, as shown in the first two examples in Table II. Thus, although CDS can cancel offset and reduce low-frequency common-mode noise, it may result in higher frequency noise. However this can be filtered by a low pass filter. If we assume that the sampling frequency $\left(f_{s}\right)$ is high enough compared to that of the noise (ensuring $\alpha>1$ ), then the conceptual relationship between the characterization factor $\alpha$ and the sampling interval can be as depicted in Fig. 7

\section{EXPERIMENTAL TEST BED AND ALGORITHM DESCRIPTION}

The experiments were carried out on an $80 \times 80$ touchscreen panel. Details of the test bed are given in Table III. The system has an operating voltage of $10 \mathrm{~V}$ and works with a refresh rate at $60 \mathrm{~Hz}$.

The common-mode noise and offsets on electrodes don't change dramatically between adjacent frames. Thus, by saving the noise pattern (i.e. treating it as a noise reference frame)

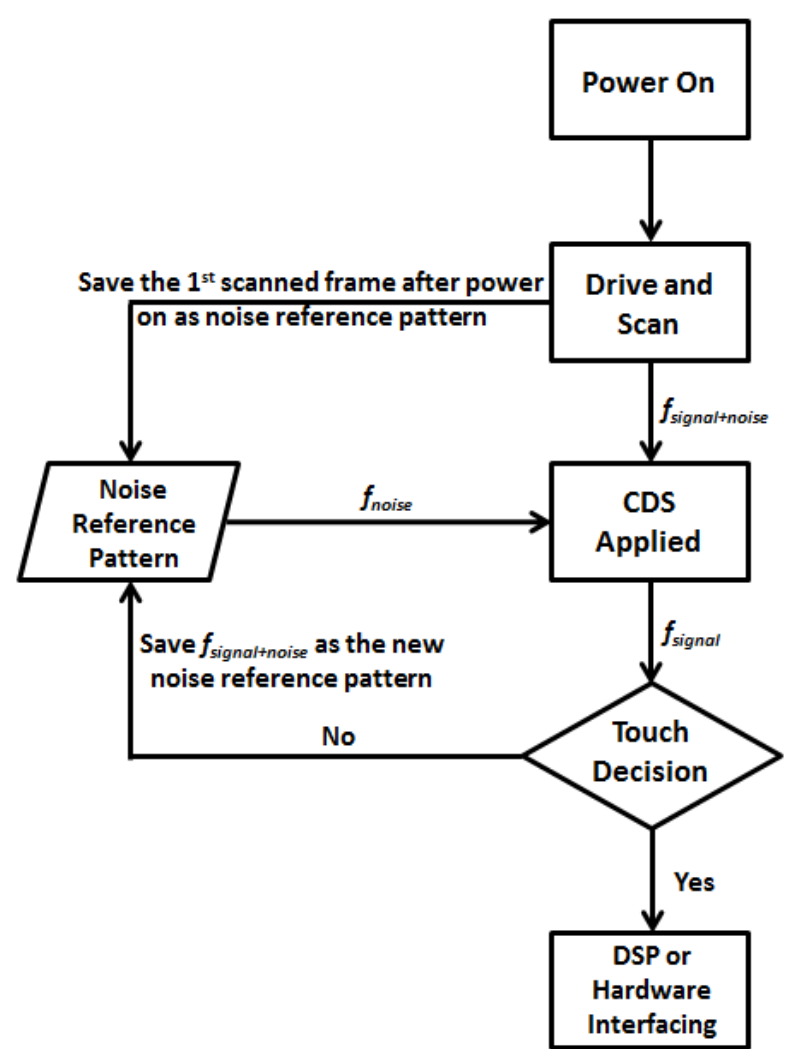

Fig. 8. Flowchart of the CDS based common-mode noise and global multi-valued offset reduction method.

which is constantly updated as shown in Fig. 8, and deducting it from the touch signal image, the low frequency and common-mode noise and offset can be canceled. The algorithm's flowchart is shown in Fig. 8. After turning the device on, the drive lines are powered individually and sense lines work in sequence to measure the intersections' voltages. The first scanned frame is the noise frame (denoted as $f_{\text {noise }}$ ), which has no touch signal but contains information of the noise and offset voltage value on the electrode. This is saved as a reference pattern. Then the system scans the panel periodically. After each scan, the retrieved data (denoted as $f_{\text {signal+noise }}$ ) is subject to the CDS algorithm, and a new frame (denoted as $f_{\text {signal }}$ ) is generated. This procedure can be expressed as:

$$
f_{\text {signal }}(x, y)=f_{\text {signal }+ \text { noise }}(x, y)-f_{\text {noise }}(x, y) \text {; }
$$

where $(x, y)$ represents the location of the electrode intersections. Then the frame $f_{\text {signal }}$ is sent to the decision function to decide if a touch has happened or not. If the frame $f_{\text {signal }}$ is interpreted as a touch event, the data will be used for further processing depending on the hardware/software environment. For example, the touch signal can be used to open a folder on desktop or close a webpage. If not, the frame $f_{\text {signal+noise (prior to applying the CDS algorithm) will be saved as }}$ the new or replacement noise reference pattern. In this way, the noise reference pattern can be updated automatically with the most recent noise information. 


\section{V.RESULTS AND DISCUSSION}

As explained above, CDS can efficiently cancel the global multi-valued offset as well as reduce the low-frequency common-mode noise. In the experiments, two separate sets of data from sampling frequencies of $30 \mathrm{~Hz}$ and $60 \mathrm{~Hz}$ are used. Examples of applying CDS to single and multi-touch related touch images are illustrated in Fig. 9. The normalized output (no touch event) PSD plots are used to analyze the effect of CDS. Based on Fig. 10, before CDS a strong DC component is observed due to the offset. In Fig. 10 (a), we observe that when below $8 \mathrm{~Hz}$, after applying CDS with the $60 \mathrm{~Hz}$ sampling frequency, the noise power drops. But between $9 \mathrm{~Hz}$ to $17 \mathrm{~Hz}$, the noise power is of a similar level as without CDS. Above $18 \mathrm{~Hz}$, the noise power increases. When the sampling frequency is down to $30 \mathrm{~Hz}$, only below $3 \mathrm{~Hz}$ does the noise power drop after CDS. This is because the correlation of low-frequency noise between adjacent frames is relatively weak at low sampling frequencies. This correlation increases at higher sampling frequencies at the expense of energy consumption. Since the offsets behave as DC, they can be canceled regardless of sampling frequency.

As mentioned earlier in Section III, CDS introduces higher frequency noise within a certain bandwidth. This is related to the sampling frequency and explained by the example below. Consider a noise component with frequency $f_{s} / 2$, which is subject to CDS of frequency $f_{s}$. The distance between the two adjacent sampling points is $\pi$. Thus after CDS, the output $y$ is expressed as

$$
\begin{aligned}
y & =A \sin \left(2 \pi \frac{f_{s}}{2} t+\varphi+\pi\right)-A \sin \left(2 \pi \frac{f_{s}}{2} t+\varphi\right) \\
& =-2 A \sin \left(\pi f_{s} t+\varphi\right)
\end{aligned}
$$

where $A$ and $\varphi$ are the amplitude and the initial phase of the waveform, respectively, $t$ is the time to sample the waveform. This indicates that the absolute output is doubled after CDS. Similar analysis can be made for other sampling frequencies.

As the touch signal has a low frequency property, the

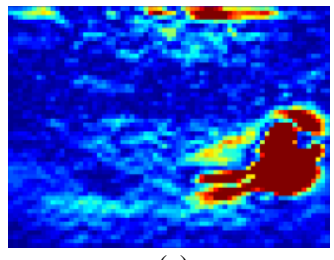

(a)

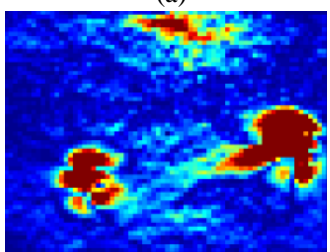

(c)

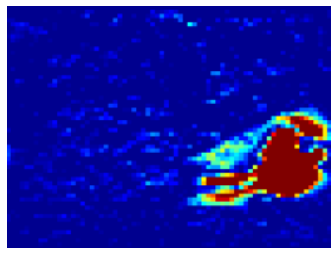

(b)

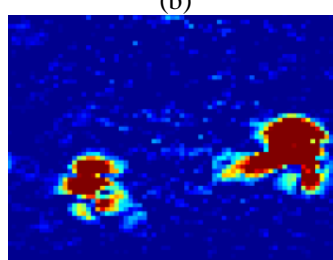

(d)
Fig. 9. The touch related images before and after CDS. (a) and (c) are raw images based on one and two touch events. (b) and (d) are the CDS processed images

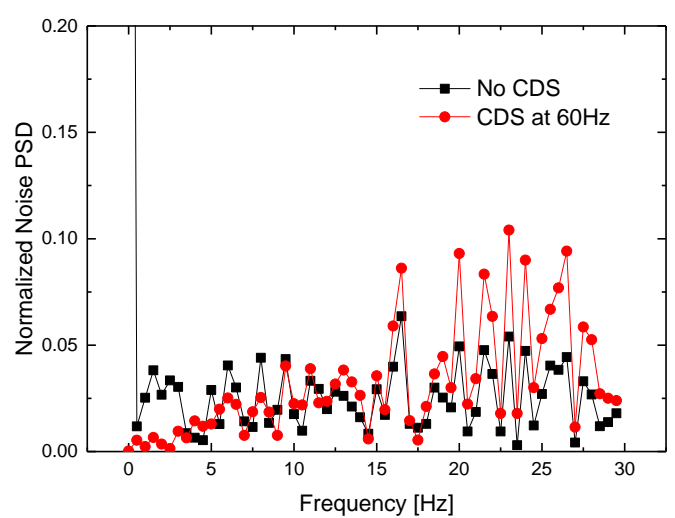

(a)

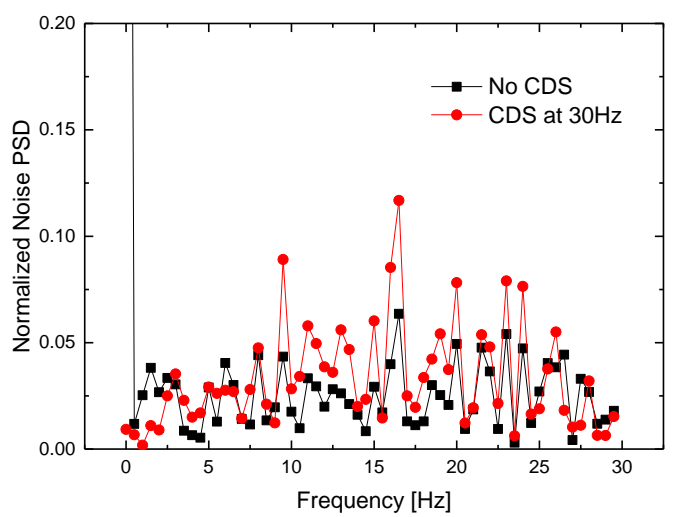

(b)

Fig. 10. Normalized PSD plots of the original output and CDS outputs with sampling frequency at (a) $60 \mathrm{~Hz}$ and (b) $30 \mathrm{~Hz}$.

increased high frequency noise power can be filtered. Within $10 \%$ of $f_{s}$, SNR is boosted by $5.9 \mathrm{~dB}$ and $7.6 \mathrm{~dB}$ when sampling frequencies are $30 \mathrm{~Hz}$ and $60 \mathrm{~Hz}$, respectively. Beyond this frequency, the SNR starts degrading. Thus CDS is powerful in reducing common-mode noise, and is expected to further enhance the SNR in the TSPs summarized in Table I.

\section{Algorithm Robustness}

The assignment of the most recent frame that does not generate a touch event to be the noise reference pattern might raise the concern that an unexpected 'bad frame' resulting from a temporary hardware malfunction might result in a deterioration of SNR in subsequent frames. This can be addressed by applying exponential smoothing:

$$
f_{\text {noise }}^{(n+1)}(x, y)=\alpha f_{\text {noise }}^{(n)}(x, y)+(1-\alpha) f_{\text {signal }+ \text { noise }}^{(n)}(x, y) ;(5)
$$

The weighting factor $\alpha$ can be adjusted to optimize system's performance. However, it should be noticed that this will slightly weaken the correlation between adjacent frames. 


\section{Algorithm Time Budget}

Although CDS provides significant reduction of the common-mode noise and offset, the computational time and energy consumption of the algorithm are key considerations. As with many mainstream touchscreen systems [41], the system's scan rate is $60 \mathrm{~Hz}$, which implies that the computational time should not be longer than $16.7 \mathrm{~ms}$. The computational time depends on the algorithm's complexity, which is $O(N)$ in this work. Here $N$ is the number of pixels to be processed. In the case considered here, $N$ equals 6400 . The processors in many of the current PDAs have a computational ability in the range of GHz. For example, a $1 \mathrm{GHz}$ processor would take $\sim 6.4 \mu$ s for the CDS algorithm, which is a much smaller time period compared to the system's refresh interval.

\section{Algorithm Energy Budget}

Current touchscreen controllers scan at $75 \mathrm{kHz}$ with a power consumption of $2.5 \mathrm{~mW}$ [42]. The energy $E$ for measuring each electrode intersection is

$$
E=P / f_{s}
$$

where $P$ and $f_{s}$ denote controller's power consumption and scan frequency, respectively. Thus, $1 / 30 \mu \mathrm{J}$ is needed for reading each electrode intersection. For a sensing matrix with $\mathrm{M}$ rows and $\mathrm{N}$ columns, the total power $\left(P_{\text {total }}\right)$ for scanning the whole panel can be expressed as

$$
P_{\text {total }}=(E M N) f_{s}
$$

Current commercial TSPs in mobile phones use 9 column electrodes and 16 row electrodes, which yield 144 electrode intersections. Thus the power consumption for measuring the whole panel once is $0.288 \mathrm{~mW}$ (assuming a scan rate at $60 \mathrm{~Hz}$ ), and the energy consumption for scanning each frame is $4.8 \mu \mathrm{J}$.

Current embedded processors have a power efficiency $(\eta)$ of over 20MIPS/mW [43]. Thus the power consumption $\mathrm{E}_{\mathrm{CDS}}$ for the algorithm

$$
E_{C D S}=N / \eta
$$

is roughly $7.2 \mathrm{nW}$, and the computation time is $0.14 \mu \mathrm{s}$. Therefore the energy consumption is around $1 \mathrm{fJ}$, which is much smaller than that of the touchscreen controller, and can thus be considered negligible.

\section{CONCLUSION}

The noise in touchscreen systems introduces detection errors, which lead to "fake" touch or mis-registration of touch location. In addition to that, the noise and global multi-valued offset result in high power consumption. In this paper, the touch related frame is treated as an image and the correlated double sampling algorithm is applied. Experimental results show that a good SNR enhancement of $7.6 \mathrm{~dB}$ is achieved, and the low-frequency common-mode noise and global multivalued offsets are reduced after CDS. Although CDS introduces noise within a certain bandwidth depending on the sampling frequency, it can be filtered so as not to overlap with the touch signal frequencies.

\section{REFERENCES}

[1] NPD Display Search company, "Quarterly touch panel market analysis", 2014, [Online]. Available:

http://www.displaysearch.com/cps/rde/xchg/displaysearch/hs.xsl/touch_ panel_market_analysis.asp

[2] J. Lee, M. T. Cole, J. C. S. Lai and A. Nathan, "An analysis of electrode patterns in capacitive touch screen panels", IEEE J. Display Technol., vol. 10, no.5, pp.362-366. May 2014.

[3] C. Jr. William, H. S. George, "Discriminating contact sensor", U.S. Patent 3911215 A, Oct. 1975.

[4] G. Barrett and R. Omote, "Projected-capacitive touch technology", Inform. Display Mag., vol. 26-3, pp. 16-21, 2010.

[5] A. Nathan, J. C. S. Lai, S. Cha, and C. Church, "Pressure sensing display device", U.S. Patent US20140008203 A1, Jan. 9, 2014

[6] H. Shin, S. Ko, H. Jang, I. Yun, and K. Lee, "A 55dB SNR with $240 \mathrm{~Hz}$ frame scan rate mutual capacitor $30 \times 24$ touch-screen panel read-out IC using code-division multiple sensing technique", IEEE ISSCC Dig. Tech. Papers, pp. 388-389, 2013.

[7] N. Miura, S. Dosho, S. Takay, D. Fujimoto, T. Kiriyama, H. Tezuka, T. Miki, H. Yanagawa, and M. Nagata, "A $1 \mathrm{~mm}$-pitch $80 \times 80$-channel $322 \mathrm{~Hz}$-frame-rate touch sensor with two-step dual-mode capacitance scan”, ISSCC, MEMs, and Displays, pp. 216-217, 2014.

[8] Y. Kim, and T. H. Ahmed, "Low energy and latency touch detection using group testing", EUSIPCO, pp. 1-5, 2013.

[9] S. Hotelling, J. Strickon and B. Huppi, "Multipoint touchscreen", U.S. Patent, US20060097991 A1, May 2006.

[10] Z. Baharav and R. Kakarala, "Capacitive touch sensing: Signal and image algorithms", Proc. SPIE Conf. on Comput. Imaging, Feb. 2011.

[11] M. G. A. Mohamed, U. Jang, I, Seo, H. Kim, T. Cho, H. K. Chang and S. Lee, "Efficient algorithm for accurate touch detection of large touch screen panels", IEEE ISCE, pp. 1-2, Jun. 2014.

[12] M. H. White, and D. R. Lampe, "Characterization of surface channel CCD image arrays at low light levels", IEEE J. Solid-State Circuits, vol. sc-9, pp. 1-13, Feb. 1974.

[13] C. Enz, G. Temes, "Circuit Techniques for Reducing the Effects of Op-Amp Imperfections: Autozeroing, Correlated Double Sampling, and Chopper Stabilization", in Proc. IEEE, vol. 84, no. 11, Nov. 1996.

[14] J. Ohta, "Smart CMOS image sensors and applications", CRC Press, 2007.

[15] Y. Ahn, T. Ahn, K. Lee and J. Kang, "Avoiding noise frequency interference with binary phase pulse driving and CDS for capacitive TSP controller", IEICE Electronics Express, vol. 11, no. 21, pp. 1-7, Oct. 2014.

[16] V. Souchkov, "Capacitive touchscreen signal acquisition without panel reset", U.S. Patent US8274491 B2, Sep. 2012.

[17] D. Lim, S. Lee, W. Choi, J. Park and D. Jeong, "A digital readout IC with digital offset canceller for capacitive sensors", J. Semicond. Tech. Sci., vol. 12, no. 3, Sep. 2012.

[18] J. Park, D. Lim and D. Jeong, "A $6.3 \mathrm{~mW}$ high-SNR frame-rate scalable touch screen panel readout IC with column-parallel $\Sigma-\triangle \mathrm{ADC}$ structure for mobile devices", IEEE A-SSCC, pp. 357-360, Nov. 2013.

[19] R. J. Lowles, J. A. Robinson, K. Wu, "System and method of integrating a touchscreen within an LCD”, U.S. Patent US7388571 B2, Jun. 2008.

[20] J. H. Yang, S. C. Jung, Y. J. Woo, J. Y. Jeon, S. W. Lee, C. B. Park, H. S. Kim, S. T. Ryu and G. H. Cho, "A novel readout IC with high noise immunity for charge-based touch screen panels", IEEE CICC, pp. 1-4, Sep. 2010.

[21] A. Middleton, B. Rella, H. J. Kim, M. Baily, A. Coleman and G. Garavuso, "FTIR multi-touch screen technology", Rochester Institute of Technology, Project No. P12302, 2012.

[22] S. Aras, T. Nihei and A. Rahman, "Touch sensing method and apparatus", U.S. Patent 20130015867 A1, Jan. 2011.

[23] O. Nys, H. Ballan, N. Chappatte and F. Vuadens, "Circuit for capacitive touch applications”, U.S. Patent, US20110001492 A1, Jan. 2011.

[24] H. Ballan, N. Chappatte, O. Nys and F. Vuadens, "Circuit for capacitive touch applications", W.O. Patent WO2012034714 A1, Mar. 2012. 
[25] Y. Guedon, K. B. Tan, K. A. Ningrat and D. Guo, "Capacitive sensing analog front end”, U.S. Patent, US20110242048 A1, Oct. 2011.

[26] Cypress Semiconductor Corp. "Using correlated double sampling to reduce offset, drift and low frequency noise", [Online]. Available: http://www.cypress.com/?rID=2894

[27] O. Korobeynikov, J. M. Kulinets, V. Protasov and P. R. Nuykens, "Methods and apparatus for reducing non-ideal effects in correlated double sampling compensated circuits", U.S. Patent US20090273392 A1, Nov. 2009.

[28] J. Bussat and S. P. Hotelling, "Redundant sensing element sampling", U.S. Patent US20140015774 A1, Jan. 2014

[29] J. B. Roberts, "Baselining technologies in force-based touch panel systems", U.S. Patent US20030210235 A1, Nov. 2003.

[30] C. J. Brown, H. Kato, K. Maeda and B. Hadwen, "A continuous-gain silicon-system LCD with optical input function", IEEE J. Solid-State Circuits, vol. 42, no. 12, pp. 2904-2912, Dec. 2007.

[31] I. Yang and O. Kwon, "A touch controller using differential sensing method for on-cell capacitive touch screen panel systems", IEEE Trans. Consum. Electron., vol. 57, issue 3, pp. 1027-1032, Aug. 2011.

[32] O. Kyong and H. Lim, "Photo sensing circuits using low-temperature polycrystalline silicon TFTs and photo diode for smart functional displays", SID Symposium Digest of Technical Papers, vol. 41, issue 1, pp. 684-687, May 2010.

[33] J. Lee, D. Yeo, J. Um, E. Song, J. Sim, H. Park, S. Seo, M. Shin, D. Cha and H. Lee, "A 10-touch capacitive-touch sensor circuit with the time-domain input-noise isolation", SID Symposium Digest of Technical Papers, vol. 43, issue 1, pp. 493-496, Jun., 2012.

[34] 3M company, "Touch technology brief: Projected capacitive technology", US, 2011.

[35] J. Yang, S. Park, J. Choi, H. Kim, C. B. Park, S. Ryu and G. Cho, “A highly noise-immune touch controller using Filtered -Delta-Integration and a charge-interpolation technique for 10.1-inch capacitive touch-screen panels", IEEE ISSCC Dig. Tech. Papers, pp. 390-391, Feb. 2013.

[36] H. Jang, H. Shin, S. Ko, I. Yun, and K. Lee, "2D coded-aperture-based ultra-compact capacitive touch-screen controller with 40 reconfigurable channels", IEEE ISSCC Dig. Tech. Papers, pp.218-219, Feb. 2014

[37] S. Ko, H. Shin, J. Lee, H. Jang, B. C. So, I. Yun, and K. Lee, "Low noise capacitive sensor for multi-touch mobile handset's applications", IEEE ASSCC, Nov. 2010, pp. 1-4.

[38] S. Ko, H. Shin, H. Jang, L. Yun, and K. Lee, "A 70 dB SNR capacitive touch screen panel readout IC using capacitor-less trans-impedance amplifier and coded Orthogonal Frequency-Division Multiple Sensing scheme", IEEE Symposium on VLSIC, pp. C216-C217, 2013.

[39] J. E. Park, D. H. Lin, and D. K. Jeong, "A reconfigurable 40-to-67 dB, 50-to-6400 Hz frame-rate, column-parallel readout IC for capacitive touch-screen panels", IEEE J. Solid-State Circuits, vol. 49, no. 10, Oct. 2014.

[40] K. Kim, S. Byun, Y. Choi, J. Baek, H. Cho, J. Park, H. Ahn, C. Lee, M. Cho, J. Lee, S. Kin, H. Kwon, Y. Choi, H. Na, J. Park, Y. Shin, K. Jang, G. Hwang and M. Lee, "A capacitive touch controller robust to display noise for ultrathin touch screen displays", IEEE ISSCC, Medical, Displays and Imagers, pp. 116 - 117, Feb. 2012.

[41] T. Marks II, (2013). Touchscreen latencies in flagship tablets. (Available:http://appglimpse.com/blog/touchmarks-ii-touchscreen-laten cies-in-agship-tablets/)

[42] C. Luo, M. A. Bokar, A. J. Redfern, and J. H. McClellan, "Compressive sensing for sparse touch detection on capacitive touch screens", IEEE Trans. Emerg. Sel. Topics Circuits Syst., vol. 2, no. 3, pp. 639-648, Sep. 2012.

[43] ARM, "Cortex-a5 processor performance", [Online]. Available: http://www.arm.com/products/processors/cortex-a/cortex-a5.php

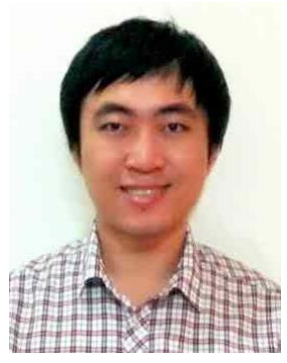

Shuo Gao received the M.Sc. in electrical engineering from the University of Ottawa, Canada, 2013. He is currently a Ph.D. student in the Hetero-Genesys Laboratory, University of Cambridge. Previously he worked as an optical fiber system engineer at Ciena Corporation, Ottawa, Canada. His research interests include touch interactivity and RF system for flexible electronics.

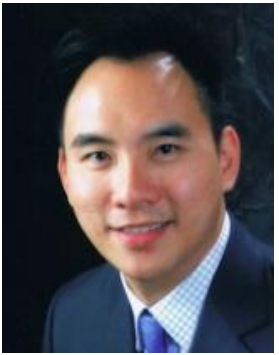

Jackson Lai received the Ph.D. in electrical engineering from the University of Waterloo, Canada, in 2007. He is currently a Senior Display Technology Developer at Blackberry. Previously, he has held positions such as Device and Circuit Engineer in Carestream Health, as well as Lead Display Circuit Designer in Ignis Innovation Inc. His research interests include imaging circuits, large area electronics, as well as system on panel design.

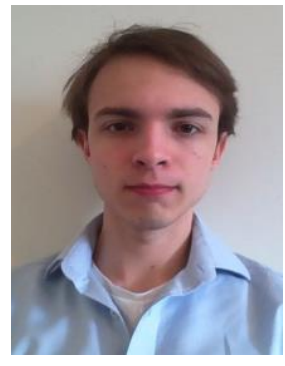

Charles Micou is an Engineering student at the University of Cambridge, U.K., in the final year of his M. Eng degree. After two years of general engineering, he specialised into Electrical and Information Sciences. His areas of interest include Computer Vision, Machine Learning, Image Processing, and Embedded Systems. He has worked as a software engineer on projects ranging from embedded telecommunications devices at Eseye, to cloud-based distributed simulations at Improbable.

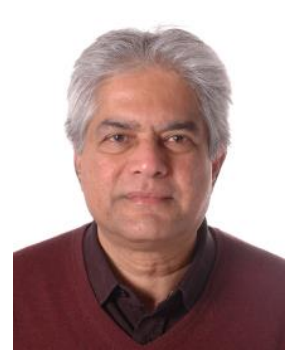

Arokia Nathan (S'84-M'87-SM'99-F'10) received the Ph.D. degree in electrical engineering from the University of Alberta. Following post-doctoral years at LSI Logic Corp., USA, and ETH Zurich, Switzerland, he joined the University of Waterloo, Canada, where he held the DALSA/NSERC Industrial Research Chair in sensor technology and subsequently the Canada Research Chair in nano-scale flexible circuits. He was a recipient of the 2001 NSERC E.W.R. Steacie Fellowship. In 2006, he moved to the U.K. to take up the Sumitomo Chair of Nanotechnology at the London Centre for Nanotechnology, University College London, where he received the Royal Society Wolfson Research Merit Award. He has held Visiting Professor appointments at the Physical Electronics Laboratory, ETH Zürich and the Engineering Department, Cambridge University, U.K. He holds the Chair of Photonic Systems and Displays in the Department of Engineering, Cambridge University. He has published over 500 papers in the field of sensor technology, $\mathrm{CAD}$, thin film transistor electronics, and is a co-author of four books. He has over 50 patents filed/awarded and has founded/co-founded four spin-off companies. He serves on technical committees and editorial boards in various capacities. Dr. Nathan is a Chartered Engineer (U.K.), Fellow of the Institution of Engineering and Technology (UK), Fellow of IEEE (USA), and an IEEE/EDS Distinguished Lecture. 